\title{
Needle Deflection and Sewability on Lockstitch Sewing Machine
}

\author{
Part 1. Evaluation Method of Needle Deflection
}

\author{
By Michi Tanaka*, Kuniko Yasumori* and Yoshinobu Kamata**,Members,TMSJ \\ * Kawamura Junior College \\ **Faculty of Education, Kagawa University
}

Based on the Journal of the Textile Machinery Society of Japan, Vol.48,No.3,T70- T77(1995- 3)

\begin{abstract}
It is established that in sewing operation, a needle could be deflected by factor such as sewn fabric structure. Empirically, however, this deflection is considered to be closely related to sewability, but without defining the proper correlation they may have. A series of studies are, therefore, undertaken to find out the correlation between sewabiliity and needle deflection in sewing operation. The present paper is aimed at a proposed method for evaluating the needle deflection. In the method, the deflect-ing force is measured by two gauges attached to the lateral surfaces of a needle shank, and then the deflection is estimated by using a simple needle model. Some model studies are carried out on synthetic paper and cotton fabrics, and their results are presented.
\end{abstract}

\section{Introduction}

In apparel industry, the preparatory processes of sewing, e.g., plan of making patterns, setting up of designs, etc., are important, but the process of sewing itself is more important because the quality finishing of a cloth is generally decided by the performance of sewing process. It may be believed that the performance of sewing process will depend upon both the sewn object and the sewing operation, namely operational simplicity in the sewing process, i.e., sewability.

With respect to sewability, the problems that may arise during sewing are usually tackled to a great extent by the inherent skill of an operator. However, with the recent trends in diversification of textile materials, fabrics with new material properties are being developed day by day, and consequently, the operator's skill alone is simply becoming insufficient to tackle the cloth sewability problems. In fact, this phenomenon has also been acknowledged by many in the recent years. In this respect, therefore, the studies on sewability and the accumulation of sewability data are very essential to find out an effective solution. A systematic study to clarify the sewing mechanism in terms of needle- fabric interaction is felt to be addressed first for this purpose.

During sewing of fabrics, the occurrence of uneaven seam, local thread cutting, etc., are very common. The research works published on these phenomena are very rare. Empirically, however, these phenomena may be thought, for example, due to lateral needle deflection. In turn, the fact of needle deflection may thought to be closely related to sewability. The results of a preliminary study conducted by our group lead to the opinion that the factors like needle shape and eccentricity, the construction of the fabric to be sewn, various operational conditions, etc., are responsible for lateral needle deflection. In line of the above, a detailed study is, therefore, formulated to find out the factors actually responsible for lateral needle deflection, and therefrom to establish a relation between those prospective factors of needle deflection and sewability. The present paper constitutes a part of the study which proposes the measuring method of lateral needle deflection along with other details citing examples.

There are many literature on the resistance that a needle may experience from fabric during sewing, but almost all are regarding the penetration/withdrawal forces on the needle. As for example, the relationship of needle penetration force and thread cutting [1], the relationship of penetration force and needle shape [2], the relationship of needle penetration force and needle temperature [3], etc., could be found in literature.

\section{Measuring method for needle deflection}

When a needle penetrates to and withdraws from cloth, it experiences resistance from the cloth and thereby deflected somewhat. In the following sections, we shall call the resistance that deflects the needle "needle deflecting force". An attempt has been made here to measure this needle deflecting force by taking the bending moment of the needle into account. For measuring this bending moment, the shank of the needle was milled to a depth of $0.3 \mathrm{~mm}$ diametrically at two places to get orthogonal planes, and then strain gauges (N11-1- 120- 11 foil strain gauge, Kyowa Co. Ltd.) were attached on those planes (Fig. 1). With respect to the strain gauge, therefore, the moment arm will change continuously as the arm shaft rotates. However, for our convenience of making the data- handling easy at latter stages, an apparent needle deflecting force was obtained at first by assuming the moment arm as a constant, and then the force was determined by considering the values of actual moment arm which in our study changes with the arm shaft rotation. As shown in Fig. 2, the $x-y$ Cartesian coordinate 


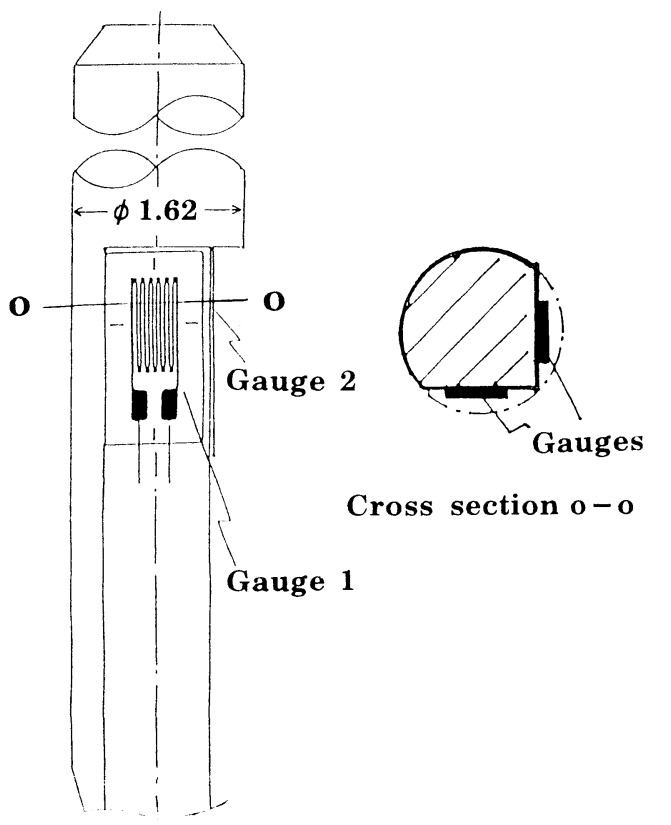

Fig. 1 Position of gauges attached to needle shank

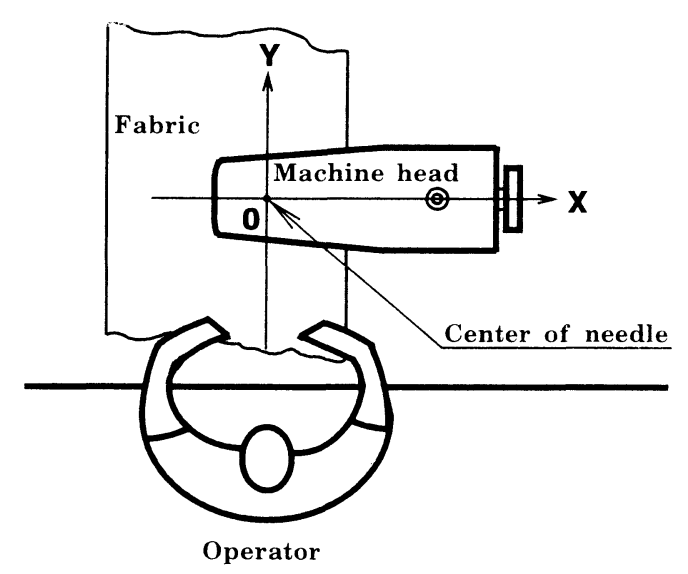

Fig. 2 X-Y coordinate system The origin :center of needle, $Y$ axis: sewing direction

system with its origin coinciding the needle axis is adopted to represent the force- displacement characteristics of needle deflection. The positive side of ordinate represents the marching direction of sewing. The force corresponding to the output of gauge \#1 which represents the force along sewing direction is denoted as $F_{y}$, and the force corresponding to the output of gauge \#2 which represents a force along perpendicular to sewing direction is denoted as $F_{x}$ ' (see Fig. 1 for the gauge position). Therefore, the forces $F_{x}$ 'and $F_{y}^{\prime}$ would be the components of apparent needle deflecting force. It is essential to mention at this point that the mutual perpendicularity of the positions of two attached gauges was confirmed by experiments in which we had checked that the application of strain in one gauge caused almost no output at the other gauge. During experiment, the detected strain was measured by a dynamic strain meter (6MB2, NEC San- ei Instruments, Ltd.), recorded by a wave recorder (Omniright 8M36, NihonDenkiSanei Co. Ltd.), and then stored in a personal computer (PC9801UV, NEC Co. Ltd.). In order to determine the arm shaft rotational angle, a marker signal was considered at the upper dead point of the needle bar.

\subsection{Wave Recorder:}

Some of the specifications of the wave recorder used in this study are as follows; memory $32 \mathrm{~kW}$, maximum continuous sampling time $6.4 \mathrm{~s}$, sampling speed $200 \mu \mathrm{s}$, etc. With this wave recorder, therefore, the data acquisitions for as low as $3.8 \mathrm{deg}$ arm shaft angular interval are possible even if the machine speed become as high as 3,200 spm. However, as the present experiments were conducted at a slower machine speed of $200 \mathrm{spm}$, the data acquisitions for a further smaller arm shaft angular interval of $0.24 \mathrm{deg}$ were realized.

\subsection{Natural frequency of needle:}

As mentioned in Table 1, a half-finished needle $(\mathrm{DB} \times 1$, \#9; Orugan Needle Co. Ltd.) was used in this study. In the experiments, this needle itself was also acted as the detector of needle deflecting force. The natural frequency of this needle was found to be $1,700 \mathrm{~Hz}$ from the free oscillation experiments. It may be seen from this value of natural frequency and the wave form of needle deflecting force in a cycle (discussed later), the data acquisitions for up to a machine speed of 34,000 spm approximately are possible. However, as the lockstitch sewing machine is generally operates at a speed of 3,000 spm, the simulated conditions of the needle detector are believed to be sufficient enough for the data acquisition. Moreover, as because the present experiments were conducted at a low sewing speed of $200 \mathrm{spm}$, the limitation of the simulated characteristics is further unlikely to occur.

\section{Data processing:}

\subsection{Evaluation of needle deflecting force:}

Let, the depth of needle- tip penetration be $z$, and the location of the upper surface of the needle plate be the starting point of this penetration, i.e., $z=0$. Then, by using the relations shown in Fig. 3, the value of $z$ could be given as

$$
z=-c-a \cdot \cos \theta+\sqrt{b^{2}-a^{2} \sin ^{2} \theta}
$$

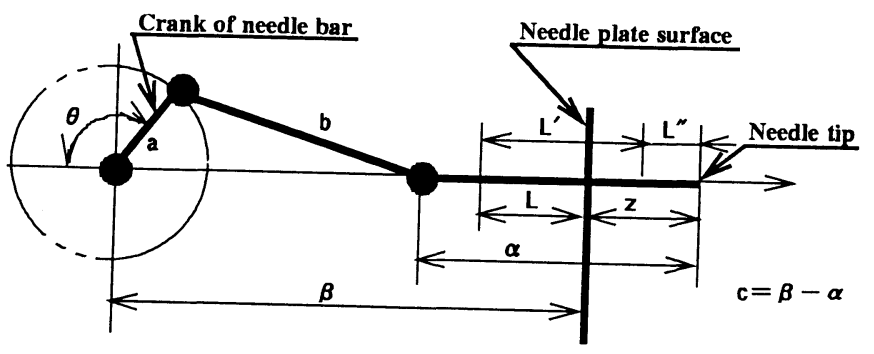

Fig. 3 Illustrated scheme of position of needle tip 
where $\theta$ is the rotating angle of the arm shaft with respect to upper dead point of the needle bar (i.e. $\theta=0$ ), and $a, b, c$ are constants. The moment arm $L(\theta)$, i.e., the distance from the upper surface of the needle plate to the mid point of the strain gauge attached to the needle is evaluated by the equation

$$
\left.\begin{array}{ll}
\mathrm{z}>0 ; & \mathrm{L}=\mathrm{L}{ }^{\prime}+\mathrm{L}^{\prime \prime}-\mathrm{z} \\
\mathrm{z} \leqq 0 ; & \mathrm{L}=0
\end{array}\right)
$$

Here, $L^{\prime \prime}$ is the distance between needle tip and needle thread eye, and $L^{\prime}$ is the distance between needle thread eye and mid point of the strain gauge attached to the needle. Now, the respective relations between the components of actual needle deflecting force, $F_{x}$ and $F_{y}$, and between the components of apparent needle deflecting force, $F_{x}^{\prime}$ and $F_{y}^{\prime}$, are

$$
\begin{aligned}
& F_{x}=F_{x}^{\prime} L^{\prime} / L \\
& \text { and } \\
& F_{y}=F_{y}{ }^{\prime} L^{\prime} / L
\end{aligned}
$$

Therefore, the resultant needle deflecting force, $F$, and its direction, $\Phi$, will be

$$
\mathrm{F}=\sqrt{\mathrm{F}_{\mathrm{x}}^{2}+\mathrm{F}_{\mathrm{y}}^{2}}
$$

and

$$
\phi=\tan ^{-1}\left(F_{y} / F_{x}\right)
$$

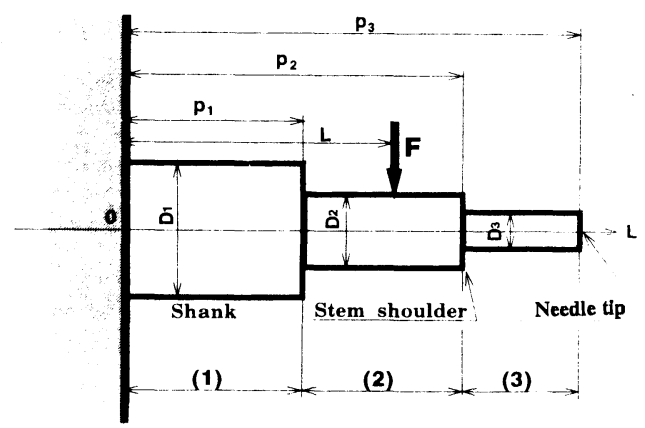

Fig. 4 Simple model needle used for evaluation of needle deflection

\subsection{Evaluation of needle- tip deflection:}

The evaluation of needle- tip deflection (hereafter called as needle deflection) is done here from the estimation of needle deflecting force. When a needle is at its penetrated condition, the cloth usually tends to move a little along the sewing direction depending upon the needle type used in the sewing. But in this study, it is simply assumed that the cloth does not move on the needle plate. Furthermore, with respect to the needle shape, it is also assumed that the shank, stem shoulder and needle head are all circular in cross- sections but different in diameters.
Therefore, for the application of needle deflecting force, F, at a designated position L (Fig. 4), the needle deflection, $\delta$, could be considered into the following three different cases.

$$
\begin{aligned}
0 \leqq & \leq \mathrm{p}_{1} \\
\delta= & \left(\mathrm{F} / 6 \mathrm{EI} I_{1}\right) \mathrm{L}^{2}\left(3 \mathrm{p}_{3}-\mathrm{L}\right) \\
\mathrm{p}_{1} \leqq & \mathrm{~L} \leqq \mathrm{p}_{2} \\
\delta= & \left(\mathrm{F} / 6 \mathrm{EI} \mathrm{I}_{1}\right) \mathrm{p}_{1}\left\{\mathrm{p}_{1}\left(3 \mathrm{p}_{3}-\mathrm{p}_{1}\right)+3\left(\mathrm{~L}-\mathrm{p}_{1}\right)\left(2 \mathrm{p}_{3}-\mathrm{p}_{1}\right)\right\} \\
& +\left(\mathrm{F} / 6 \mathrm{EI} \mathrm{I}_{2}\right)\left(\mathrm{L}-\mathrm{p}_{1}\right)^{2}\left\{3\left(\mathrm{p}_{3}-\mathrm{p}_{1}\right)-\left(\mathrm{L}-\mathrm{p}_{1}\right)\right\} \\
\mathrm{p}_{2} \leqq & \mathrm{~L} \leqq \mathrm{p}_{3} \\
\delta= & \left(\mathrm{F} / 6 \mathrm{EI} \mathrm{I}_{1}\right)\left\{3 \mathrm{p}_{1}\left(\mathrm{~L}-\mathrm{p}_{1}\right)\left(2 \mathrm{p}_{3}-\mathrm{p}_{1}\right)+\mathrm{p}_{1}^{2}\left(3 \mathrm{p}_{3}-\mathrm{p}_{1}\right)\right\} \\
& +\left(\mathrm{F} / 6 \mathrm{EI} I_{2}\right)\left[3\left(\mathrm{~L}-\mathrm{p}_{2}\right)\left(\mathrm{p}_{2}-\mathrm{p}_{1}\right)\left\{2\left(\mathrm{p}_{3}-\mathrm{p}_{1}\right)-\left(\mathrm{p}_{2}-\mathrm{p}_{1}\right)\right\}\right] \\
& \left.+\left(\mathrm{p}_{2}-\mathrm{p}_{1}\right)\left\{3\left(\mathrm{p}_{3}-\mathrm{p}_{1}\right)-\left(\mathrm{p}_{2}-\mathrm{p}_{1}\right)\right\}\right] \\
& +\left(\mathrm{F} / 6 \mathrm{EI} I_{3}\right)\left(\mathrm{L}-\mathrm{p}_{2}\right)\left\{3\left(\mathrm{p}_{3}-\mathrm{p}_{2}\right)-\left(\mathrm{L}-\mathrm{p}_{2}\right)\right\}
\end{aligned}
$$

Here, $E$ is the modulus of elasticity, and $I_{i}(i=1,2,3)$ are the moments of inertia of the respective cross- sectional areas. As the cross- sections are assumed to be circular with diameter of $D_{i}$, so $I_{i}=\pi D_{i}{ }^{4} / 64$.

\subsection{Data processing flow:}

Data collections during the experiments, and their processing sequences followed here are as stated below. By using the wave recorder, the time dependent changes of $F_{x}$ ' and $F_{y}$ ' were recorded at first. During this process, a marker signal indicating the position of upper dead point of the needle bar $(\theta=\mathbf{0})$ was also taken into account. The data were then sent to a personal computer, and stored in a floppy disc. These raw data are processed for the consecutive cycles (two adjacent marker signals represent one machine cycle). As the values of $L$ corresponding to $\theta$ are obtainable from the Equation (2), so the needle deflecting force, $F$, and its direction, $\Phi$, could be calculated from the Equations (5) and (6), respectively. Once these are obtained, the needle deflection, $\delta$, may be calculated by using the Equations (7) to (9). Therefore, the relations between $\theta-\mathbf{F}$, and $\theta-\delta$ could be found out. Moreover, as the Equation (1) may be used to transform $\theta$ into $z$, so the final relations between $z-F$, and $\mathbf{z}-\delta$ could be obtained. The results so obtained by the above processing will be shown here in the figures followed.

\section{Case studies:}

Depending on the types of sewn samples used in the experiments, two different case studies were undertaken, viz., one by using a synthetic paper and the other by using a plain woven cotton broadcloth. Some of the details of sewing machine, needle, sewn samples, etc., used in the experiments are shown in Table 1. The sewing operation was performed without sewing thread (i.e., false sewing). Again, the needle used here was an axisymetric model 
needle having no needle eye, groove and scarf. The needle was collected in this pre-finished condition from an intermediate stage of a needle production line. This is so because a finished sewing needle available commercially in the market is usually asymmetric with respect to needle axis. Owing of such asymmetry, a finished needle may get deflected on its own during the penetration into the cloth. Therefore, with the use of a model needle such as one stated above, we could avoid the problem of inherent needle deflection during penetration, and consequently, the effect of sewn material on the needle deflection may be obtained more clearly. As mentioned before, two sewn samples were used in the experiments, viz., a synthetic polypropylene paper to represent the materials having no weave structure, and a plain woven cotton broadcloth to represent the weave textured materials. Their selections were aimed at not only to measure and analyze the needle deflection, but also to establish that the present analytical method is helpful for the evaluation of needle deflection caused by weave textures.
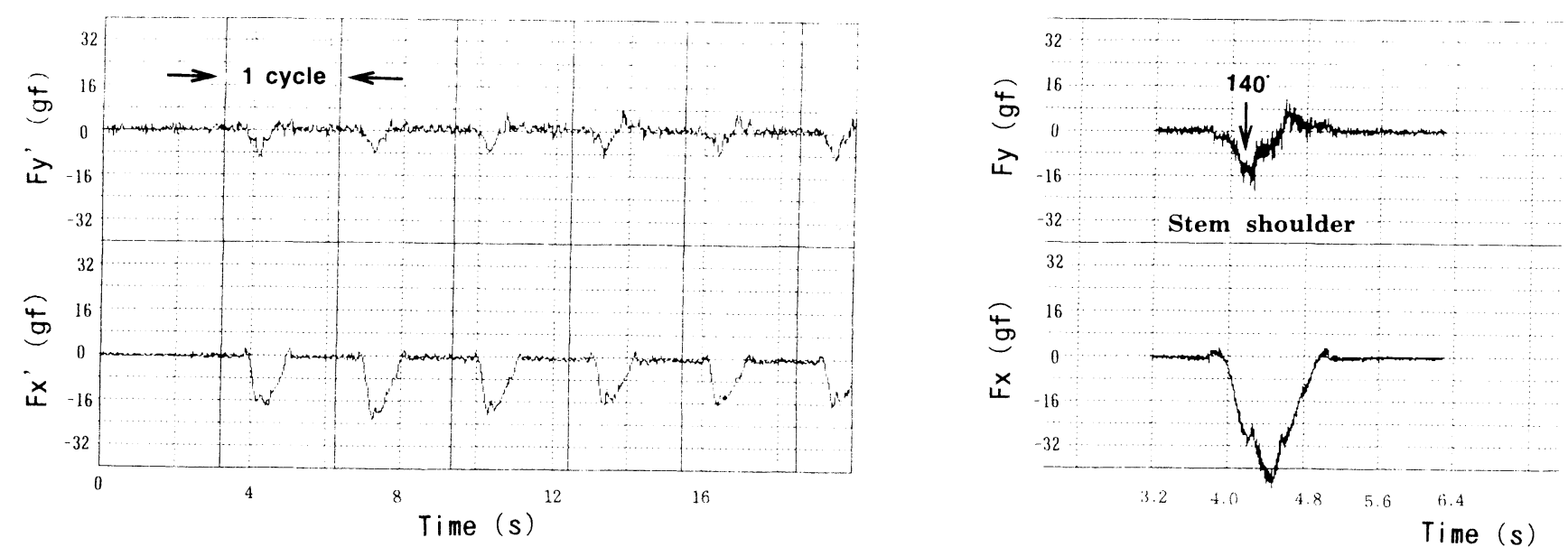

Fig. 5 A typical trace of needle deflecting force with time The force was assumed to be concentrated at position of needle eye, sewn sample: synthetic paper, sewing speed: $200 \mathrm{spm}$

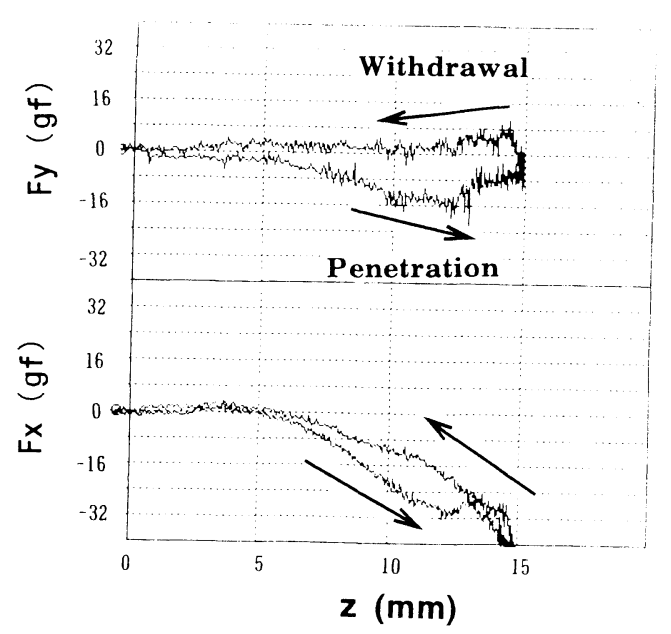

Fig. 7 Needle deflecting force with needle penetrating depth

The data are same as Fig. 6

4.1. Case study using synthetic paper:

The characteristics of needle deflection that occurred when a synthetic paper was used as sewn sample is described here along with the details of data processing.

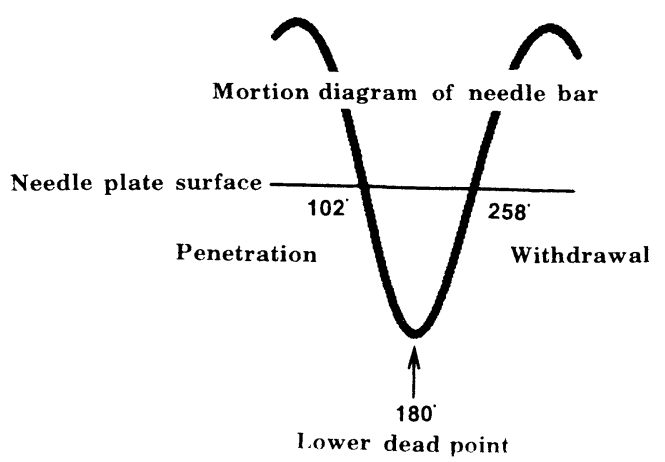

Fig. 6 Relationship between deflecting force and motion diagram of needle bar

\section{A. Experimental data:}

Figure 5 shows a typical data set in which the components of apparent needle deflecting force ( $F_{y}{ }^{\prime}$ and $\left.F_{x}{ }^{\prime}\right)$ obtained for a fixed moment arm of $\mathrm{L}^{\prime}=19.6 \mathrm{~mm}$ are shown along the vertical axis. Marker signals which correspond to the upper dead point of the needle bar $(\theta=$ $0)$ are also shown in the figure by solid vertical lines. Good reproducibility in the wave form for each stitch (each cycle) may be noticed. Therefore, it may be said that with respect to needle penetration, the structure of synthetic paper is quite uniform.

\section{B. Analysis of wave form:}

A detail of the data analysis for a stitch corresponding to the left- most wave form of Fig. 5 is presented here. 
Taking actual moment arm into account, the components of apparent needle deflecting force of the wave form are transformed into the components of actual needle deflecting force $\left(F_{x}\right.$ and $\left.F_{y}\right)$ by using the Equations (3) and (4). These are shown in Fig. 6 along with the corresponding relationship with the motion diagram of needle bar calculated by the Equation (1). Based on their relationship with the needle bar motion diagram, the variations of needle deflecting force components against the corrected needle penetration depths are shown in Fig. 7. For calculations, the values used in the Equations (1) and (2) are as follows; $\mathrm{a}=15.3 \mathrm{~mm}, \mathrm{~b}=48.5 \mathrm{~mm}, \mathrm{c}=49.3$ $\mathrm{mm}, \mathrm{L}^{\prime}=19.2 \mathrm{~mm}$, and $\mathrm{L}^{\prime \prime}=3.3 \mathrm{~mm}$.

It may be seen from Fig. 6 that the needle begins to penetrate the cloth at $\theta=102 \mathrm{deg}$, reaches to the lower dead point at $\theta=180 \mathrm{deg}$, and completes withdrawing at $\theta=258 \mathrm{deg}$. Consequently, the needle deflecting force is observed for a period of $102 \mathrm{deg}-258 \mathrm{deg}$ only. Moreover, the variation in needle deflecting force, if any, due to penetration of needle stem shoulder into the cloth may also be observed because the latter event occurs at about $\theta$ $=140 \mathrm{deg}$ which is well within the range of $\theta$ we studied. As shown in Fig. 7, the needle deflecting force increases gradually up to a position corresponding to the
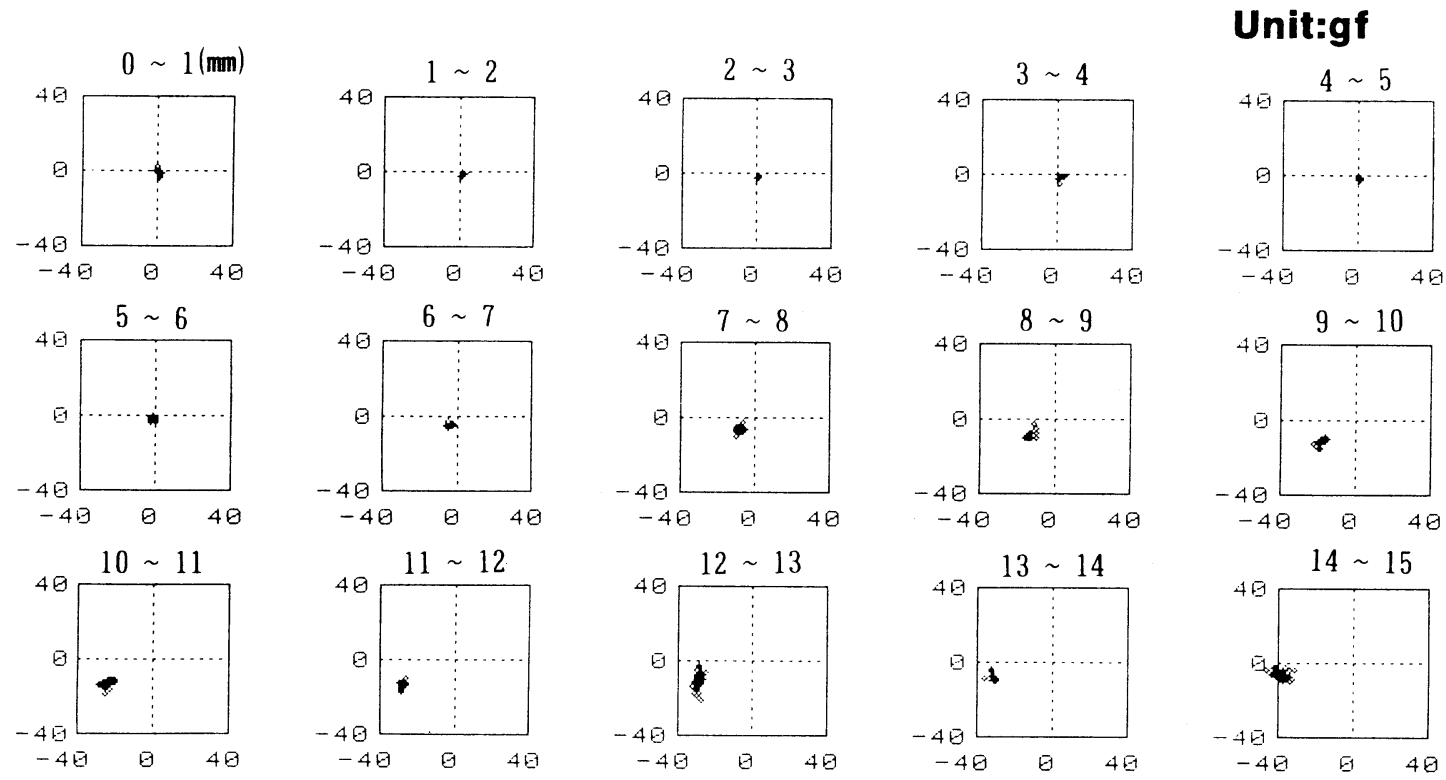

Fig. 8 Dimension and direction of needle deflecting force when the penetrates (synthetic paper) The data are same as Fig. 6

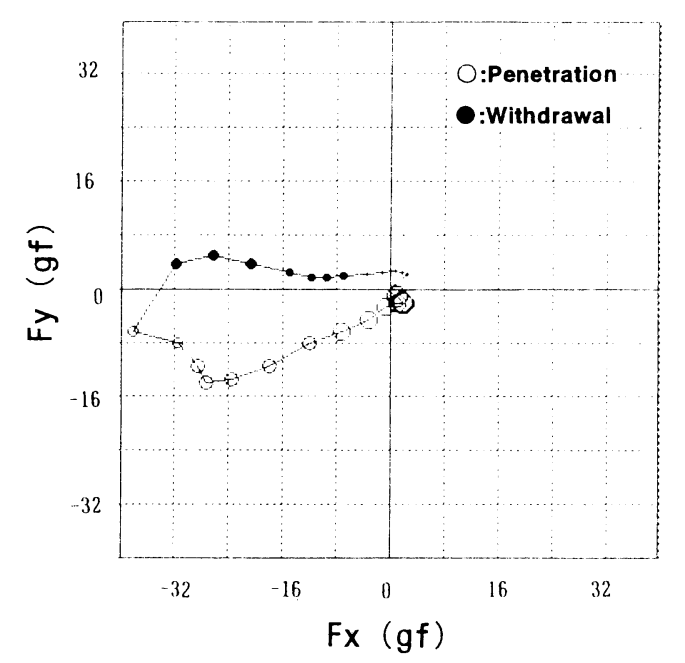

Fig. 9 Continuous variation of needle deflecting force per cycle when the needle penetrates and withdraws (synthetic paper)

The data are same as Fig. 6

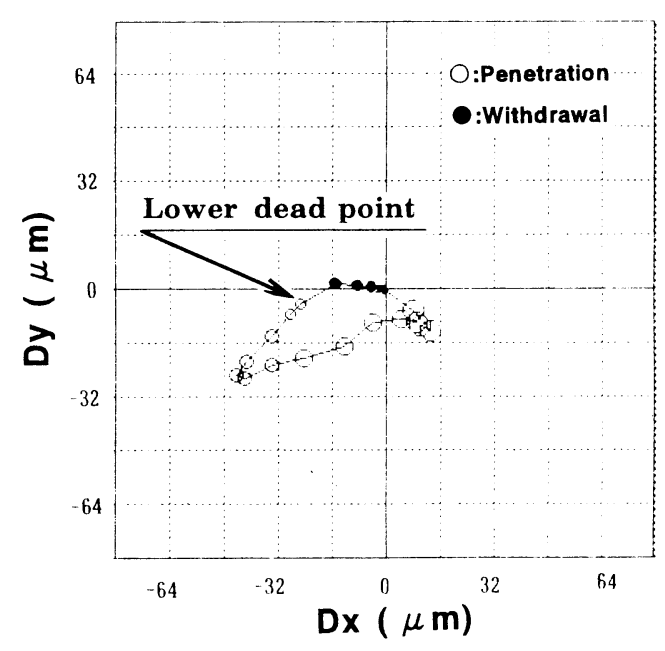

Fig. 10 Continuous variation of needle deflection per cycle when the needle penetrates and withdraws (synthetic paper)

The data are same as Fig. 6 
penetration of needle stem shoulder and then decreases quickly up to a position where the needle enters its withdrawal stage. This decrease may be believed to be due to the structural failure of the sewn sample. Figure 8 shows fifteen different plots corresponding to every $1 \mathrm{~mm}$ penetration depth starting from the point of needle tip penetration to the point where needle-tip reaches the lower dead point. The center of each plot denotes the position of needle drop, and the scattered points inside the plot represent the data (dimension and direction of needle deflecting force) acquired during that depth of penetration. The plot corresponding to the position of $12-13 \mathrm{~mm}$ is believed to be for the penetration of needle stem shoulder, and the one corresponding to $14-15 \mathrm{~mm}$ position is for the location where the needle reaches to its lower dead point. The needle deflecting forces developed during these two positions notably show abrupt variations as compared to other needle positions. Likewise, therefore, the dimension and direction of needle deflecting force developed during needle penetration may be understood by studying the states of the plots of pause- feed frames.

A systematic attempt is made now to understand the details of the changes occur in needle deflecting force and needle deflection during the courses of penetration and withdrawal. As for example, it may be seen clearly from the data of needle deflecting force in Fig. 6 that the occurrence of friction between needle and cloth is also takes place during the development of needle deflecting force. Consequently, the considerable variation seen in the measured values may thought to be due to presence of such friction. Moreover, the presence of electrical noise may also be seen. Because of such mingling, therefore, it is difficult to see all the aspects if individual data is used for expression. In this study, therefore, a modified way of expression using average of motions for every $1 \mathrm{~mm}$ penetration depth, like the one shown in Fig. 8, is adopted as a method of applying filter. The needle deflecting forces calculated in accordance with the above approach are collectively shown in Table 2 . In addition, the needle deflection, $\delta$, calculated according to the method described in Section 3.2 and the direction of needle deflection, $\delta$, calculated therefrom are also shown in this Table. As the modulus of elasticity of steel in longitudinal direction ranges from 20.1-21.6 $\mathrm{Nm}^{-2}$ [4], a value corresponding to the average of lower and upper limits, namely $E=2.1 \times 10^{7} \mathrm{gf} / \mathrm{mm}$ is considered as the longitudinal elastic modulus of needle. The continuous variations of the needle deflecting forces for both penetration and withdrawal stages are calculated by using the results of Table 2, and are shown in Fig. 9. The corresponding variations of the needle deflections are shown in Fig. 10. From these two figures, it is evident that the cyclic forms of needle deflecting force and needle deflection are different though their directions are same. This may be due to the non- matching of the order of respective magnitudes. In the present example, the maximum needle deflecting force and the maximum needle deflection are estimated as $39 \mathrm{gf}$ and $52 \mu \mathrm{m}$, respectively. By graphical means, therefore, the needle deflecting force which a needle receives from the cloth during sewing, and also the needle deflection, could be estimated. In other words, the in-plane force which a cloth receives from the needle will be known.

Table 2 An exsample of needle deflection when the needle penetrates the paper

1) Sewing machine: JUKI DDL 5580

Sewing speed: $200 \mathrm{spm}$

Bar pressure: $4.5 \mathrm{kgf}$

Hight of feed dog: $0.85 \mathrm{~mm}$

Stitch length: $2 \mathrm{~mm}$

3) Sewing thread:

Not used

1. Synthetic paper (Yupo TGB \#90)

Weight per unit area: $89.1 \mathrm{gf} / \mathrm{cm}^{2}$

2. Cotton broadcloth ( $\# 80)$

Density: 55 ends $/ \mathrm{cm}, 30$ picks $/ \mathrm{cm}$

Thickness: $0.22 \mathrm{~mm}$

Weight per unit area: $1.17 \times 10^{-2} \mathrm{gf} / \mathrm{cm}^{2}$ Twist: Type of $S$ for both Warp and Weft Twist Angle: Warp is $9^{\circ}$ and Weft $23^{\circ}$

5) Sewing direction: Parallel to the direction of warp

6) Number of sample layers: 1 ply.

7) Needle: Half- finished needle without needle eye, groove and scarf, and \#9 regular point.

\section{Needle deflection:}

As described above, the values of needle deflection, and thereby the maximum needle deflection, for each stitch cycle could be obtained from the analysis of wave form in question. Figure 11 shows the accumulation of the values of maximum needle deflection for 45 consecutive cycles. The magnitudes of the values of maximum needle deflection are within the range of 20- $30 \mu \mathrm{m}$ approximately, and almost all are positioned in one direction collectively. It may be wrong to think that whatsoever variations are 
there in the above results are due to the quality of synthetic paper because the reproducibility of experimental data with synthetic paper is very good (Fig. 5). It may be thought, however, that a small eccentricity at the needle-tip be responsible for these results. This is

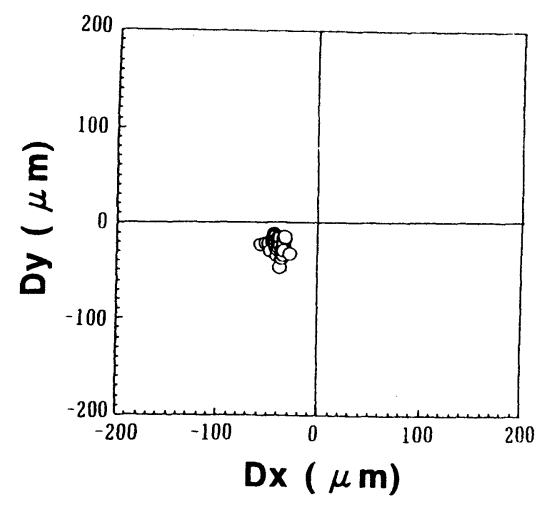

Fig. 11 Scatter of maximum deflection with synthetic paper

Sewing speed: $200 \mathrm{spm}$, Number of stitches: 45 because, in general, the needle- tip is not necessarily align with the needle axis accurately even though we wanted to chose such a model needle. The details of the effect of needle eccentricity on the needle deflection will be published in a separate report.

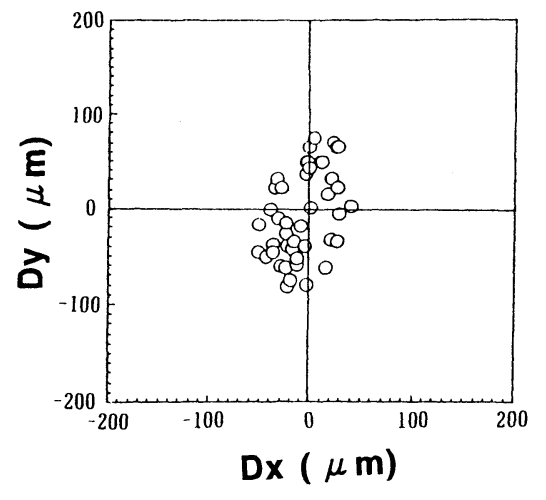

Fig. 13 Scatter of maximum deflection with cotton plain fabric Sewing speed: $200 \mathrm{spm}$, Number of stitches: 45

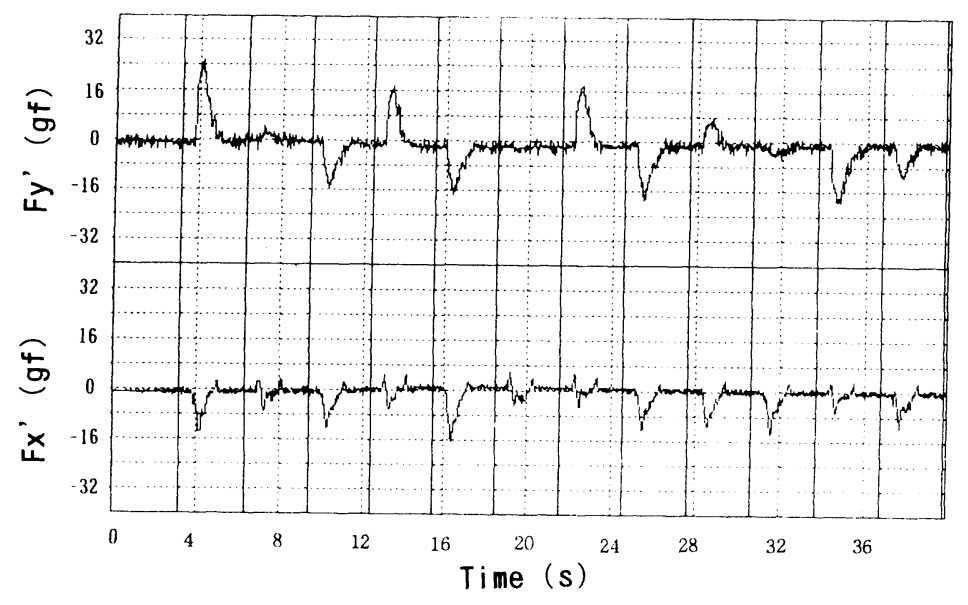

Fig. 12 A typical trace of needle deflecting force with time The force was assumed to be concentrated at position of needle eye, sample fabric: Cotton plain weave, sewing speed: $200 \mathrm{spm}$

4.2. Case study using plain woven cotton broadcloth: Experimental data obtained for the case when a plain woven cotton broadcloth was used as sewn sample are shown in Fig. 12. Upon comparison with the test results obtained for the case of synthetic paper (Fig. 5), it may be seen that the wave form of each stitch cycle differs considerably from the other. It seems that this fact originates from the constructional properties of the cloth. It may be suggested that the needle- tip is caught at by the weaving threads during penetration into the cloth. In Fig. 13, the accumulations of the values of maximum needle deflection for 45 consecutive cycles are shown. If we compare these with the results obtained for the case of synthetic paper (Fig. 11), it may be understood that the needle is certainly deflected by the texture of the cloth. Furthermore, as many of the data are positioned in the direction same as one for the case of synthetic paper, the effect of needle eccentricity is thought to be included in the test results. The extent of the effect of needle eccentricity is, however, difficult to define at this stage because the effect of cloth texture is also included. Nevertheless, it is clearly observed that the cloth texture influences the needle deflection. The details of the relationship between cloth texture and needle deflection will be published in a separate paper.

\section{Conclusions:}


After establishing a measurement system for needle deflection, the evaluation method for the same is presented with an aim of better understanding of the phenomena of needle deflection, and deflecting force that a sewing needle experiences during penetration to and/or withdrawal from the cloth. Therefore, it may be believed that the finding of a relationship between these results and cloth sewability would be easy in future. As for the next program, a detailed study is planned to find out the correlation between needle deflection and other factors, e.g., needle eccentricity, weave structure, sewing direction, etc., and then to establish the relationship between those results and cloth sewability.

\section{Acknowledgment:}

The authors gratefully acknowledge the support of
M/s Orugan Needle Co. Ltd. for supplying the model needle used in the present study, and the considerable assistance of Dr. Ashoke K. karmokar with regard to the translation.

\section{Literature cited}

[1] See, for example, N.Ikegami, M.Kurita, T.Hasegawa and M.Kawarai; J. Text. Mach.Soc. Japan, 31, P103(1978)

[2] See, for example,Y.Kamata, S.Tsunematsu, R.Kinoshita and S.Naka; Sen'i Gakkaishi, 33, T157(1977)

[3] M.Onoue, U.Kawai and Y.Kamata; Sen’i Gakkaishi, 45, 542(1989)

[4] Chronological Table of Science(A Desk Type), Maruzen, (444)(1992) 